\title{
Linking microbial community analysis and ecosystem studies: A rapid lipid analysis protocol for high throughput
}

\author{
Teri C. Balser ${ }^{1,2^{\star}}$, Chao Liang ${ }^{3}$, Jessica L. M. Gutknecht ${ }^{4}$ \\ 1 Department of Soil Science, University of Wisconsin-Madison, 1525 Observatory Drive, Madison, WI 53706, USA \\ 2 Faculty of Science and Engineering, Curtin University, Perth, Western Australia, 6845 Australia \\ 3 Institute of Applied Ecology, Chinese Academy of Sciences, Shenyang 110016, China \\ 4 Department of Soil, Water, and Climate, University of Minnesota, 439 Borlaug Hall, 1991 Upper Buford Circle, St. Paul, MN 55108, USA
}

\section{ARTICLE INFO}

Article history:

Received May 26, 2018

Revised September 6, 2018

Accepted September 12, 2018

Keywords:

Microbial community composition

Lipid analysis

PLFA

MIDI-FAME

Ecosystem studies

\begin{abstract}
A B S TR A C T
During the past two decades interest in linking soil microbial community composition and activity with ecosystem scale field studies of nutrient cycling or plant community response to disturbances has grown. Despite its importance there are challenges in making this linkage. Foremost is the question of analytical feasibility. In general, microbiological community-level methodologies have not been readily adaptable to the large sample sizes necessary for ecosystem-scale research. As a result, it has been difficult to generate compatible microbial and ecosystem data sets. Soil lipid analysis shows potential as a middle ground between simple biomass measures and molecular profiling. However, the two protocols that have most often been followed are either rapid but indiscriminate (total lipid analysis or fatty acid methyl ester analysis; FAME), or precise but time consuming (phospholipid fatty acid analysis; PLFA). In this paper we report results from a standardized soil used test a modified extraction method (the 'hybrid' method) developed to balance the speed of FAME and the precision of PLFA in order to increase sample throughput. In comparing the three methods, we find that FAME and PLFA are qualitatively and quantitatively distinct. The FAME method yielded the highest fatty acid abundance, but also had high variance resulting in low precision. The PLFA method had precision, but low yield. The 'hybrid' method fell midway between FAME and PLFA for quantitative fatty acid yield. In addition, the hybrid extraction can be completed in a fraction of the time it takes for PLFA. The hybrid protocol appears to provide an optimal balance between effort and accuracy and therefore is a good choice for large-scale ecosystem studies.
\end{abstract}

(C) Higher Education Press 2019

\section{Introduction}

It is becoming increasingly clear that inclusion of microbiological information in long-term and large-scale ecosystem studies may be necessary to achieve the goal of increasing our understanding of, and ability to predict, ecosystem response to environmental and anthropogenic change (Cavi-

\footnotetext{
* Corresponding author

E-mail address: teri.balser@curtin.edu.au (T.C. Balser)
}

gelli and Robertson, 2000; Zak et al., 2003; Schimel, 2004; Balser and Firestone, 2005; Fraterrigo et al., 2006; Balser et al., 2010; Gutknecht et al., 2012). There is growing evidence that the traditional paradigm of microbial ecology, "everything is everywhere and the environment selects" (Baas-Becking, 1934), may not hold true, requiring a broader view for microbial ecology studies (Balser and Wixon, 2009; Wallenstein and Hall, 2012). However, despite growing demand for microbiological data that can be incorporated into ecosystem models, the practical and conceptual mechanisms for linking microbial detail and ecosystem-scale studies remain subjects of debate. This is due in part to the challenges inherent in 
microbial community study; microbiological communities are exceedingly complex and their structure and function are difficult to quantify, let alone connect (Balser et al., 2002; Jackson et al., 2007). Microbial ecology studies relying on DNA or RNA based techniques can provide highly detailed community-taxonomic data sets with qualitative information about microbial functional capabilities. There is a need though in large-scale ecosystem ecology studies to more broadly parameterize the microbial community in a way that can be related to soil processes (Balser and Firestone, 2005; Fraterrigo et al., 2006; Cusack et al., 2011; Jesus et al., 2016,) but to undertake this kind of approach has difficulties. Ecosystem-scale studies typically consist of sample sizes numbering in the hundreds, in contrast to sample sizes of approximately $80-200$ that are more typical of a microbial ecology study. Molecular characterization of microbial communities can be time consuming and often yields data that are not quantitative or compatible with typical ecosystem metrics (e.g. gene sequence data cannot easily be related to nitrogen mineralization rates). To accurately draw linkages between belowground community structure and processes observable at the ecosystem scale, there is a need for methods that offer a high throughput, accurate quantification of soil microbial community characteristics.

Analysis and quantification of soil lipids has seen increased use in ecosystem studies as a means to address these needs (Ben-David et al., 2011; Frostegård et al., 2011; Fichtner et al., 2014; Willers et al., 2015a; Willers et al., 2015b). Because the abundance of individual fatty acids varies among organisms in soil, fatty acid profiles provide a rough community 'fingerprint' and can also be used to determine the presence and relative abundance of coarse ecological groupings (such as Gram$\mathrm{Gram}^{+}$, actinobacteria, and fungi) in soil communities (Frostegård and Bååth, 1996; Drenovsky et al., 2004; Balser and Firestone, 2005). Additionally, because membrane lipids turn over rapidly during metabolism and are quickly degraded following cell death, the concentration of lipids in soil is proportional to viable biomass (Vestal and White, 1989; Frostegård and Bååth, 1996). While lacking the taxonomic resolution of molecular analysis, lipid analysis provides a quantitative measure of microbial subgroups and biomass that can be argued is 'necessary and sufficient' for most largescale studies (Tiedje et al., 1999).

Despite its utility, lipid analysis as typically performed (using phospholipid fatty-acid analysis; PLFA) can be prohibitive for many ecosystem ecologists in terms of time and materials (Buyer and Sasser, 2012). With PLFA, cell membrane phospholipids are extracted, purified and identified using a two-phase aqueous-organic extraction (Bligh and Dyer, 1959) (The 'modified Bligh and Dyer' method often cited) followed by chromatography to separate lipids by polarity (Fig. 1). Alkaline methanolysis converts polar lipids (phospholipids) to methylester derivatives that are volatile and can be identified by gas chromatography (GC) (Vestal and White, 1989). With this method, a typical laboratory throughput is $30-40$ samples per week.

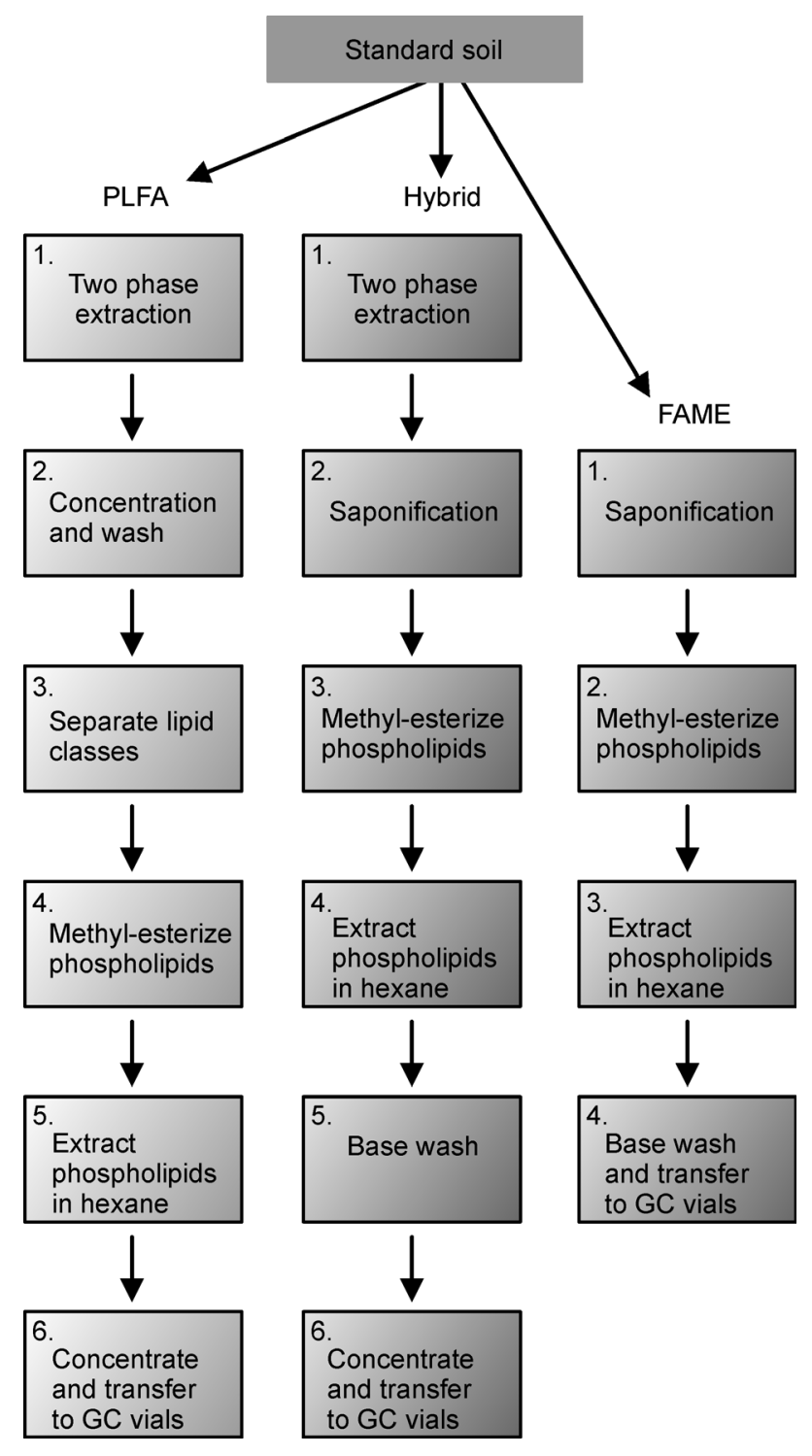

Fig. 1 Flow chart demonstrating the steps of the traditional PLFA method, the Hybrid method, and the FAME method, respectively from left to right.

An alternate method, the fatty acid methyl-ester (FAME) procedure was introduced by Microbial ID, Inc. (Newark, CA) in 1990. With FAME analysis, all ester-linked lipids are extracted directly from samples (Haack et al., 1994; Schutter and Dick, 2000;Drenovsky et al., 2004). Because it has none of the concentration or purification steps that PLFA has, the FAME method has lower overall lipid loss, and is rapid allowing well over 100 samples to be processed in the time it takes to process $30-40$ samples via PLFA. However, while the FAME method is quick, it can include lipid-like compounds coextracted from soil organic matter that distort the community signature (Nielsen and Petersen, 2000; Schutter and Dick, 2000; Jandl et al., 2005; Fernandes et al., 2013; Miura et al., 
2017). Because this inclusion can also distort biomass measures, FAME has typically been used only to coarsely qualitatively describe soil lipids (Cavigelli et al., 1995) and should be used with caution (Fernandes et al., 2013).

Despite its potential disadvantages, the idea of using FAME to characterize environmental samples (e.g., soil and sediment) is attractive for many reasons. The FAME protocol has fewer steps, requires less glassware and specialized equipment, and has a simpler experimental set-up. In addition, the greater throughput allowed by the FAME has potential to allow processing of sample numbers equivalent to most large-scale ecosystem studies. However, because the potential coextraction of soil organic matter, and thus subsequent profile distortion is a serious concern, researchers have continued to utilize PLFA despite its longer processing time and added complexity (Nielsen and Petersen, 2000; Bailey et al., 2002; Drenovsky et al., 2004; Balser and Firestone, 2005; Liang et al., 2008; Balser and Wixon, 2009; Herman et al., 2012; Schmidt et al., 2015; Kotas et al., 2018).

The primary purpose of this study was to test the feasibility and efficacy of hybridizing the PLFA and FAME protocols, to utilize the strengths of each while addressing the problem of background contamination in FAME and the slowness of PLFA. The extraction protocol combines steps from both the PLFA and FAME (Fig. 1). By performing the two-phase initial extraction and thus isolating the organic-soluble components (e.g., lipids) prior to performing FAME, this 'Hybrid' protocol offers a balance between speed and precision. Although there have been recent suggestions for modifications to both of these methods, in order to reduce hazardous chemicals, or possibly increase throughput (Willers et al., 2015b), the method described here consists of the most commonly used
Bligh and Dyer (1959) and FAME methods. This 'Hybrid' method has been used repeatedly and successfully in numerous large ecological studies (Smithwick et al., 2005; Kao-Kniffin and Balser, 2007; Gutknecht et al., 2012; Liang et al., 2015; Pei et al., 2016). The method was developed some time ago, and has been in common use for more than 15 years. Given the prevalence of its use, the recent renewal of interest in high throughput methods, and inquiries into its development, we felt that it was important to provide a report on the method's efficacy. This paper presents the results from two experimental assessments designed to 1) compare three protocols for fatty acid yield and type; and 2) test the importance of various steps in the later part of the protocol (extraction, purification, and final concentration) (Fig. 2).

\section{Materials and methods}

\subsection{Soil}

A homogenized mixture of grassland and forest soils from California, Puerto Rico, and Alaska was used as a 'standardized soil' containing diverse lipids (Table 1). This soil was created to serve as a quality control for lipid analysis in the Balser laboratory, and was included in all analytical runs. The source soils were thoroughly mixed, and roots and rocks were removed by hand. Our goal in this study was not to test discriminatory power among soils, but rather to test the impact of varying methodological steps without the confounding influence of soil variability, and to fully assess the potential of the hybrid protocol relative to PLFA and FAME for characterizing a given sample.

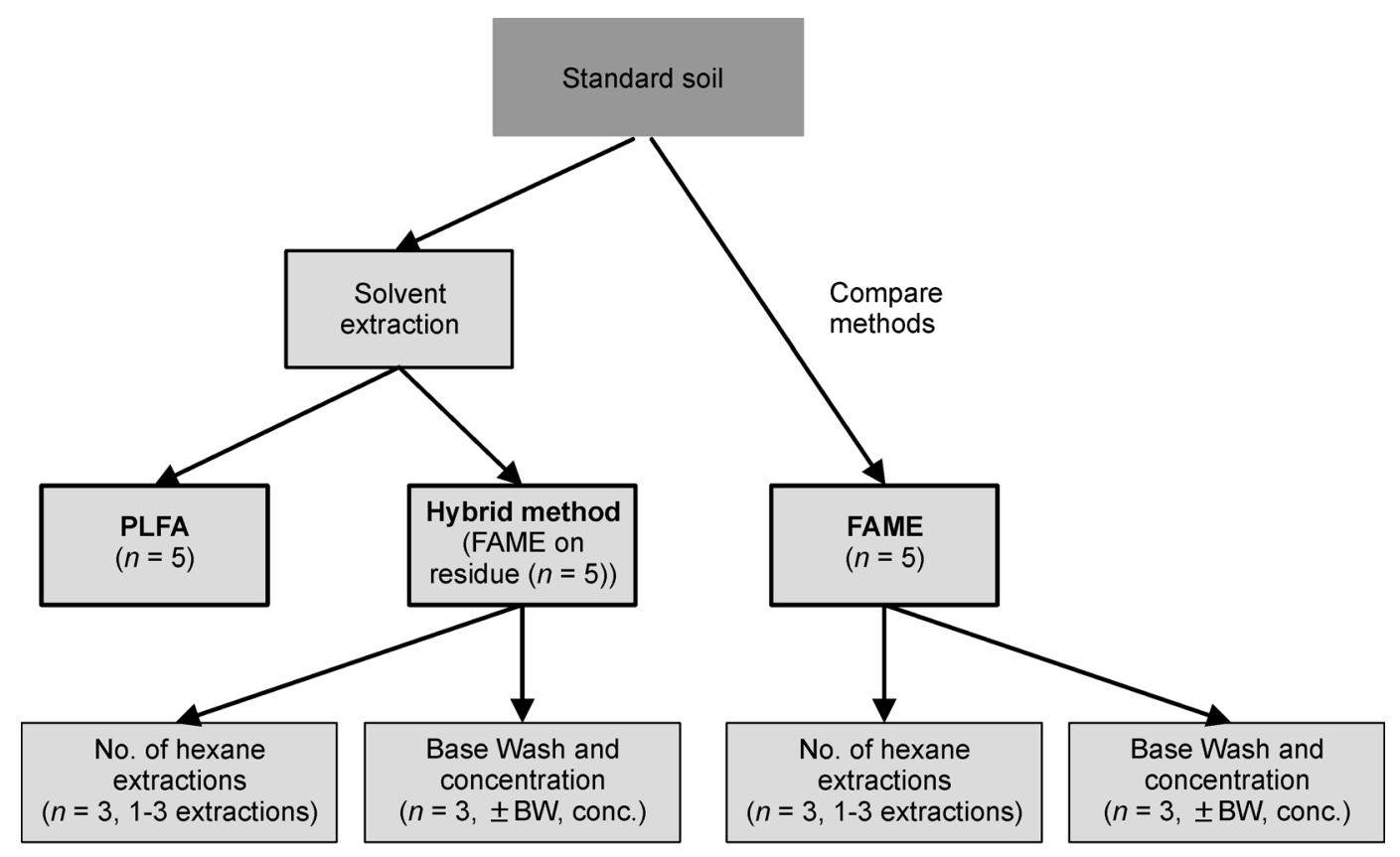

Test protocol details

Fig. 2 Outline of experimental tests performed on the Hybrid and FAME methods. Abbreviations: BW = base wash; conc. = concentration. 
Table 1 Soil properties for the standard soil used for method testing.

\begin{tabular}{lll}
\hline $\mathrm{pH}$ & & 4.9 \\
\hline$\% \mathrm{C}$ & 8.1 \\
$\% \mathrm{~N}$ & 0.26 \\
Texture (\%) & & \\
& & 64 \\
& Sand & 24 \\
& Silt & 12 \\
& Clay & \\
Minerals (ppm) & & \\
& & 396 \\
& $\mathrm{P}$ & 4330 \\
& $\mathrm{~K}$ & 3948 \\
& $\mathrm{Ca}$ & 7251 \\
& $\mathrm{Mg}$ & 269 \\
& $\mathrm{~S}$ & 70 \\
& $\mathrm{Zn}$ & $<3$ \\
& $\mathrm{~B}$ & 516 \\
& $\mathrm{Mn}$ & 30325 \\
& $\mathrm{Fe}$ & 61 \\
& $\mathrm{Cu}$ & 25487 \\
& $\mathrm{Al}$ & 180 \\
$\mathrm{Na}$ & \\
\hline
\end{tabular}

All analyses were performed by the University of Wisconsin Soil and Plant Analysis Laboratory.

\subsection{Lipid analysis: protocol descriptions}

\subsubsection{PLFA protocol}

For the PLFA protocol, we extracted, purified, and identified phospholipid fatty acids from microbial cell membranes using a modified Bligh and Dyer (1959) technique as described in Balser and Firestone (2005). In brief, we extracted $5 \mathrm{~g}$ subsamples of lyophilized whole-soil in a one-phase extraction mixture containing chloroform:methanol:phosphate buffer $(1: 2: 0.8 \mathrm{v} / \mathrm{v} / \mathrm{v})$. After the initial one-phase extraction, additional chloroform and phosphate buffer were added to create separation of the aqueous and organic phases, which were allowed to stand overnight before removal of the organic phase into a clean baked collection tube. We then separated the phospholipids from neutral and glycolipids contained in the original chloroform phase using solid phase extraction (SPE) columns containing $0.5 \mathrm{~g}$ silica gel (Supelco, Inc. Bellefonte PA) that were eluted successively with chloroform (to elute neutral lipids), acetone (to elute glycolipids), and methanol (to elute polar phospholipids). After separation and collection, polar lipids were transesterified by mild alkaline methanolysis and extracted with hexane. In a final step, samples were concentrated under nitrogen gas before analysis. We analyzed samples using a Hewlett Packard 6890 Gas Chromatograph with a $25 \mathrm{~m} \times 0.2 \mathrm{~mm} \times 0.33 \mu \mathrm{m}$ Ultra 2 (5\%-phenyl)-methylpolysiloxane column (Hewlett Packard).

\subsubsection{FAME protocol}

For the FAME protocol we followed the method of Schutter and Dick (2000), and that described by Microbial ID, Inc. (Newark CA) with modifications to test the importance of some of the intermediate steps in the protocol (Figs. 1 and 2). Modifications are described below. Briefly, the FAME method consists of four steps: saponification, methylation, extraction and base-wash. We weighed one gram of lyophilized wholesoil into ultra-clean (baked at $475^{\circ} \mathrm{C}$ for $4 \mathrm{~h}$ ) glass screw-cap test tubes and added $1 \mathrm{~mL} 3.75 \mathrm{M} \mathrm{NaOH}$ in $\mathrm{MeOH}: \mathrm{H}_{2} \mathrm{O}(1: 1$ $\mathrm{v}: \mathrm{v})$. Tubes were placed in a $100^{\circ} \mathrm{C}$ water bath for $25 \mathrm{~min}$, during which lipids present in the sample were saponified (fatty acid chains cleaved from lipids and converted to sodium salts). To convert saponified fatty acids to methyl-esters (FAMEs), a strong acid methylation was performed by adding $2 \mathrm{~mL}$ of $6.0 \mathrm{M} \mathrm{HCl}: \mathrm{MeOH}(1: 0.85)$ to each sample and incubating in a water bath at $85^{\circ} \mathrm{C}$ for $10 \mathrm{~min}$. FAMEs were extracted three times from the acidic to an organic phase by adding $1.25 \mathrm{~mL}$ of hexane:methyl tert-butyl ether (1:1), shaking the tubes for $10 \mathrm{~min}$, and removing the upper organic solvent phase to a clean baked collection tube. Centrifugation for $3 \mathrm{~min}$ at $2000 \mathrm{rpm}$ was then used to separate the phases. We transferred the organic phase to a 13 - by $100-\mathrm{mm}$ ultraclean glass Teflon lined screw cap tube. The organic phase was washed of residual acidic reagents by adding approximately $3 \mathrm{~mL}$ of mild base $(0.3 \mathrm{M} \mathrm{NaOH})$, followed by gentle mixing for $5 \mathrm{~min}$. Finally, we transferred the organic phase directly to a vial for analysis by gas chromatography. We used the same GC protocol as described above for PLFA.

\subsubsection{Hybrid protocol}

The hybrid protocol tested here combines steps from both FAME and PLFA methods (Fig. 1). First, soil lipids were extracted and concentrated in a two-phase aqueous organic extraction (as for PLFA), and then the organic-extracted material was saponified and processed as in the FAME procedure. In this way, physical and chemical soil 'background' is largely removed prior to FAME analysis (and wholesoil is not subjected to saponification).

\subsubsection{Specific tests- FAME versus hybrid methods}

Figure 2 summarizes the distinctions among the three methods and protocol details tested here. We first tested the importance of successive hexane extractions of FAMEs from the acidic to organic solution, and also looked at the importance of a base wash (Fig. 2). In addition, we investigated the value of concentrating the final product prior to $\mathrm{GC}$ analysis.

To do this, we took sets of replicate samples from our "standard soil" and explored the various steps in differing ways (Fig. 2). For the hexane extraction, we repeated the extraction step 0,1 , or 2 times (a total of 1-3 extractions), analyzing each successive extraction separately in order to determine the number and type of fatty acids in each. To test 
the importance of the base wash step, half the samples were washed as in the Hybrid procedure, and half were left unwashed, as after the methylation step in the PLFA procedure. Finally, samples in our test of the importance of the concentration step were concentrated by evaporation under nitrogen gas, for comparison with other samples where no concentration step was performed.

\subsection{Data analysis}

We added 19:0 (nonadecanoic methyl ester) and 10:0 (decanoic methyl ester) as internal standards to all samples prior to GC analysis to convert fatty acid peak areas first to concentration, then to mole percent. In all analyses we used only fatty acids that were present at $0.5 \mathrm{~mol}$ percent or higher.

We used ANOVA followed by Tukey's HSD test to compare the abundance of total and specific fatty acids extracted using each of the three methods, as well to assess the importance of the number of extractions, base wash and concentration steps. All statistical analyses were performed using JMP software (SAS Inc.). Figure preparations were accomplished using Sigma Plot (SYSTAT Software, Inc.).

\subsection{Nomenclature}

Polar lipids are designated by the number of carbons followed by the number of double bonds, the position of the double bond from the aliphatic end $(\omega)$ of the molecule and by the isomeric configuration of the double bond as either cis (c) or

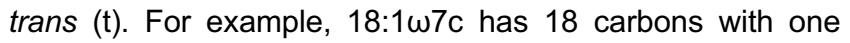
double bond at 7 carbons from the methyl end of the molecule in the cis configuration. Terminal branching is indicated by ' $\mathrm{l}$ ' (iso) or 'a' (anteiso) indicating the location of a methyl branch either one or two carbons in from the aliphatic end of the molecule (e.g. i16:0). Cyclopropyl fatty acids are given by 'cy' or 'cyclo' (e.g. 19:0 cyclo). 'Me' designates a methyl group on a given carbon inward from the hydroxyl end of the molecule (16:0 10Me has 16 carbons, no double bonds, and has a methyl group at 10 carbons from the hydroxyl end). The position of hydroxy $(\mathrm{OH})$ groups is similarly noted.

\section{Results and discussion}

\subsection{Comparing PLFA, FAME and Hybrid methods}

Our first goal with this study was to compare fatty acid yield and type across the three methods. We quantified overall yield, and the number of known and unknown fatty acids from the standard soil. 'Unknown' fatty acids are those identified as 'unknown' by MIDI-Sherlock software, or those fatty acids present in all samples with constant retention time and equivalent chain length $( \pm 0.01)$. We also looked at the relative abundance of specific lipids.

We found that the methods differed substantially in total amount of fatty acid extracted, as well as the number of known and unknown fatty acids (Fig. 3). The FAME assay resulted in the highest total extracted, followed by hybrid, then PLFA. Similarly, FAME had the highest overall number of fatty acids, as well as the highest number of unknowns; PLFA had the fewest, and the hybrid was intermediate (Fig. 3). Several research groups have compared results obtained using FAME and PLFA (Haack et al., 1994; Drijber et al., 2000; Pankhurst et al., 2001; Drenovsky et al., 2004). Their results align with our findings here. The FAME and Hybrid protocols would be expected to have higher total fatty acids extracted and the largest numbers of fatty acids, because those procedures have fewer purification steps (which tend to reduce lipid yield), and because they include glycolipids and neutral lipids in addition to phospholipids. The higher number of 'unknown' lipids in the FAME, and to a lesser degree, the hybrid method, are likely to be fatty acid-like organic materials or other
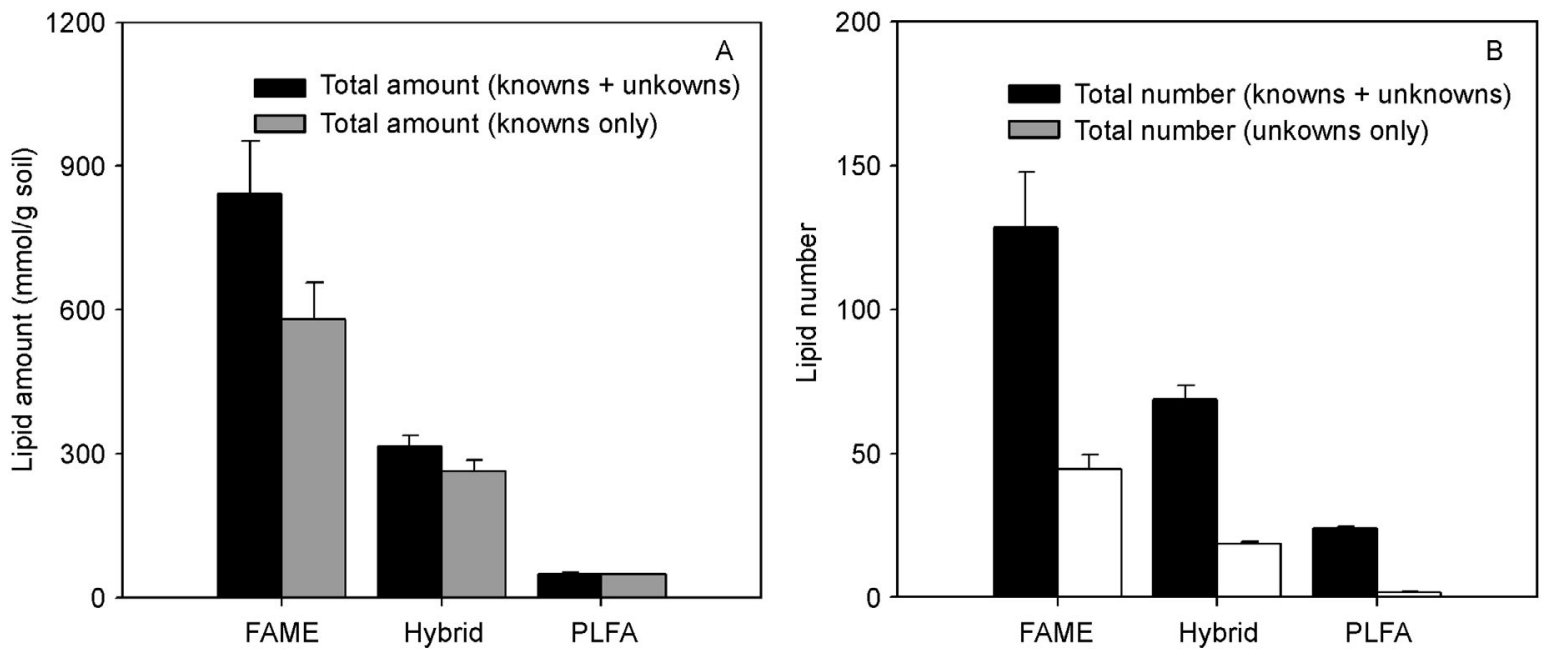

Fig. 3 Comparison of the three methods using principal components analysis on covariance matrix of known fatty acids present at $>0.5$ molar percent. Average percent coefficient of variation (cv) is given in parentheses. 
impurities resulting again from the fewer purification steps and/or from the purification effect of the silica column chromatography.

A principal components analysis (PCA) likewise indicates a difference in methods (Fig. 4). Factor analysis such as PCA is typically used to visualize differences among samples. In this case, it allows a rough comparison of the three methods and relative variability. The PLFA method has the highest average percent coefficient of variation (cv), followed by the FAME and Hybrid protocols (Fig. 4). The high cv of PLFA may be the result of low lipid yields as discussed above. The high $\mathrm{cv}$ for FAME is likely due to the "noise" in extraction - the unknown compounds that co-extract. The Hybrid method proved to be the most internally consistent of the three protocols. A Shannon index of diversity likewise indicates the paucity of final extracted compounds in PLFA (index $=0.29$ ), compared with FAME (index = 2.1). Once again, the Hybrid protocol was an intermediate, with an index of 1.0.

The PLFA method yields potentially 'cleaner' results, but it is also slower, with an extra extraction day for the lipid-class extraction step, and costly. Due to combined needs for additional reagents, stationary phase silica columns, and work hours, the totals in US dollars are \$31 per sample for PLFA versus $\$ 20$ per sample for FAME, assuming costs of high level personnel to operate the GC and cheaper labor for extractions. It is not feasible for most ecology laboratories to process the hundreds of samples in a typical ecosystem-scale study. Speed, cost, and discriminatory power may be balanced by removing the contaminating background organic matter from soils, while maintaining the speed and low cost of the FAME procedure. We believe that these points are the advantage of this hybrid protocol, extracting less soil organic material than FAME, while faster and cheaper than PLFA. This is consistent with the findings of Miura et al. (2017). They

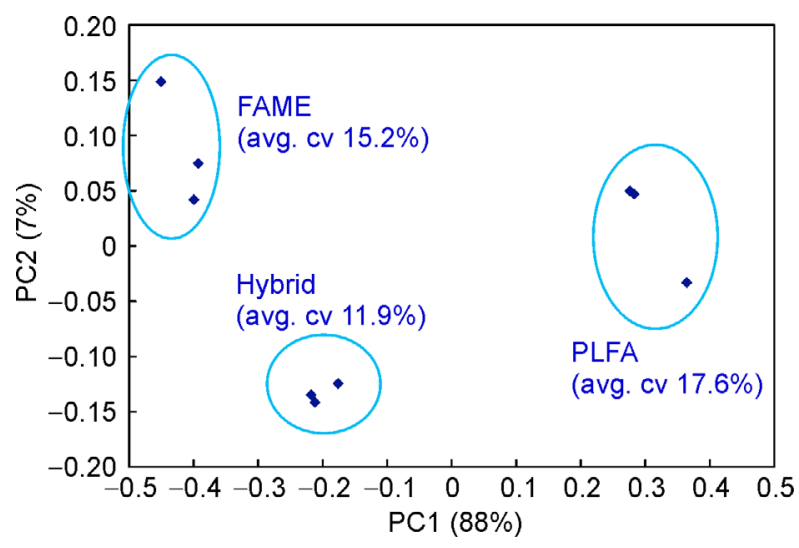

Fig. 4 Comparison of the total abundance (panel A) and number of fatty acids (panel B) extracted by PLFA, the Hybrid method, and FAME, respectively. Black bars represent the total abundance or number of lipids, gray bars (panel A) represent the total known fatty acid abundance, and white bars (panel B) represent the total number of unknown lipids extracted with each protocol. Error bars represent one standard error from the mean. compared the extraction of phospholipid fatty acids (PLFAs) to ester-linked fatty acids (EL-FAMEs) and likewise concluded that in a comparison across ecosystem types, the PLFA procedure produced a "cleaner" result, despite the greater ease of the EL-FAME. They did not test a hybrid method, as is that reported here.

Another difference among the methods was in their variability. We found that FAME yielded highly variable results, compared with PLFA (Table 2). Individual fatty acid abundances generally had higher standard deviations (S.D.) using the FAME method (Table 2), and this might lead to decreased ability to differentiate or manage different soils or draw inferences about different experimental treatments. In contrast, the PLFA method generally had very small S.D., yet resulted in only a fraction of the total fatty acid abundance. These results are not unexpected and others who have compared PLFA with FAME have reached similar conclusions (Drenovsky et al., 2004; Fernandes et al., 2013).

We also assessed the differences among methods in terms of absolute soil concentrations of fatty acids versus relative abundance. Microbial fatty acid data can be reported as an absolute amount ( $\mathrm{nmol} \mathrm{g} \mathrm{soil}{ }^{-1}$ ), or they can be reported as a relative amount (mol \% for a given fatty acid). These two ways of presenting the data portray very different aspects of microbial ecology: mol \% indicates the relative abundance of a given fatty acid in a sample and implies relative abundance change for organisms that certain fatty acids are indicative of, thus in total representing community composition change. Absolute amounts indicate biomass and actual microbial abundance change. We found differences among the three methods were clearly dependent on whether the data were calculated as absolute or relative abundance (Table 2). The FAME protocol consistently yielded higher absolute concentrations of fatty acids than PLFA, while the Hybrid method generally fell between the two. In contrast, relative abundance results were very different, and varied both individually and by lipid sub-group (Table 2). For example, the relative abundance of fatty acids with branched or cyclopropyl structures was generally highest using PLFA, while the FAME and Hybrid methods tended to yield higher relative abundances of the unsaturated groups. An exception was 18:1w7c (the precursor to lipid 19:0 cyclo), which was much higher by PLFA than Hybrid or FAME. These results could possibly be explained because of the acid chemistry of the hybrid and FAME methylation protocols compared with the alkaline chemistry of the PLFA methylation, where the differing chemistry changes the yields of different fatty acids (Zelles, 1999; Willers et al., 2015b). Finally, specific biomarker lipids also varied in relative abundance. The eukaryotic lipid $18: 3$ w6,9,12c (thought to indicate plants and higher fungi (Vestal and White, 1989) was highest by FAME, followed by Hybrid, followed by PLFA.

3.2 Assessing the importance of steps in the Hybrid and FAME methods

The second goal in this study was to assess the importance of 
Table 2 Comparison of abundance and relative abundance of fatty acids yielded from the three methods.

\begin{tabular}{|c|c|c|c|c|c|c|}
\hline & \multicolumn{3}{|c|}{ nmol fatty acid g soil ${ }^{-1}$} & \multicolumn{3}{|c|}{$\mathrm{mol} \%$} \\
\hline & FAME & Hybrid & PLFA & FAME & Hybrid & PLFA \\
\hline \multicolumn{7}{|l|}{ Saturated } \\
\hline $11: 0$ & $6.83(0.32) a$ & 5.92(0.55)a & $0(0) \mathrm{b}$ & $1.33(0.52) \mathrm{b}$ & 2.67(0.19)a & $0(0) c$ \\
\hline $12: 0$ & 14.2(0.69)a & $2.87(1.15) b$ & $0.05(0.13) \mathrm{b}$ & 2.55(0.26)a & $1.29(0.49) \mathrm{b}$ & $0.10(0.26) \mathrm{c}$ \\
\hline $14: 0$ & $1.43(0.21) a$ & $0(0) \mathrm{b}$ & $0(0) \mathrm{b}$ & $0.21(0.18) a$ & $0(0) b$ & $0(0) \mathrm{b}$ \\
\hline $16: 0$ & 72.8(1.41)a & 50.1(1.95)a & $7.98(0.64) \mathrm{b}$ & $12.7(1.14) \mathrm{c}$ & 22.6(0.89)a & $16.6(0.13) b$ \\
\hline $18: 0$ & 12.5(1.95)a & $9.76(0.27) \mathrm{a}$ & $1.76(0.23) \mathrm{b}$ & $2.16(0.16) c$ & $4.40(0.20) a$ & $3.65(0.20) \mathrm{b}$ \\
\hline \multicolumn{7}{|l|}{ Monounsaturated } \\
\hline $15: 1 \omega 8 c$ & 15.1(4.02)a & $4.55(0.14) \mathrm{b}$ & $0(0) \mathrm{b}$ & $2.65(0.25) a$ & $2.05(0.03) \mathrm{b}$ & $0(0) c$ \\
\hline $16: 1 \omega 5 c$ & 16.2(2.69)a & $6.99(0.66) \mathrm{b}$ & $1.27(0.11) \mathrm{b}$ & $2.78(0.23) a b$ & $3.15(0.23) a$ & $2.64(0.14) b$ \\
\hline $16: 1 \omega 7 c$ & 23.9(3.31)a & 10.1(0.45)b & $2.09(0.31) \mathrm{b}$ & 4.11(0.76)a & 4.54(0.09)a & $4.33(0.56) a$ \\
\hline $17: 1 \omega 7 c$ & $47.0(2.33) \mathrm{a}$ & 15.2(1.19)b & $0(0) c$ & $8.45(0.71) a$ & $6.87(0.45) \mathrm{b}$ & $0(0) c$ \\
\hline $18: 1 \omega 9 c$ & 12.1(1.00)a & 1.11(0.97)ab & $0(0) b$ & 1.75(1.52)a & $0.50(0.42) a b$ & $0(0) b$ \\
\hline $18: 1 \omega 7 c$ & 12.8(1.32)a & 8.74(0.25)a & $3.58(0.34) \mathrm{b}$ & $2.21(0.13) \mathrm{c}$ & $3.94(0.07) b$ & 7.42(0.45)a \\
\hline \multicolumn{7}{|l|}{ Branched } \\
\hline 16:0 10 methyl & 13.8(0.83)a & 9.95(0.19)b & $4.16(0.46) c$ & $2.63(0.84) c$ & $4.49(0.06) \mathrm{b}$ & 8.63(0.48)a \\
\hline $16: 0$ iso & 8.74(0.28)a & $5.36(0.15) a b$ & $2.85(0.14) \mathrm{b}$ & $1.54(0.13) \mathrm{c}$ & $2.42(0.07) b$ & 5.92(0.17)a \\
\hline $15: 0$ iso & 14.1(0.56)a & $9.08(0.52) a b$ & $5.09(0.44) \mathrm{b}$ & $2.49(0.21) \mathrm{c}$ & $4.10(0.13) b$ & $10.6(0.68) a$ \\
\hline $15: 0$ anteiso & $6.98(0.44) a$ & 4.91(0.35)ab & $2.58(0.26) \mathrm{b}$ & $1.24(0.12) \mathrm{c}$ & $2.21(0.10) \mathrm{b}$ & $5.36(0.32) a$ \\
\hline $13: 0$ anteiso & $5.27(0.21) a$ & $1.08(0.26) \mathrm{b}$ & $0(0) \mathrm{b}$ & 0.92(0.08)a & $0.49(0.11) \mathrm{b}$ & $0(0) c$ \\
\hline $17: 0$ iso & $5.33(0.27) a$ & $3.28(0.34) b$ & $1.50(0.13) \mathrm{c}$ & $0.95(0.05) c$ & $1.48(0.18) b$ & $3.11(0.14) a$ \\
\hline $17: 0$ anteiso & $4.54(0.13) a$ & $2.80(0.21) \mathrm{b}$ & $1.41(0.18) b$ & $0.81(0.08) b$ & $1.26(0.11) b$ & $2.93(0.32) \mathrm{a}$ \\
\hline 17:0 10 methyl & 13.3(14.06)a & $1.70(0.31) \mathrm{b}$ & $0.64(0.05) \mathrm{b}$ & 2.59(1.83)a & $0.77(0.15) a$ & $1.32(0.07) \mathrm{a}$ \\
\hline \multicolumn{7}{|l|}{ Miscellaneous } \\
\hline $16: 02 \mathrm{OH}$ & $4.33(1.80) a$ & $2.55(0.07) a$ & $0(0) b$ & $0.75(0.14) b$ & $1.15(0.04) a$ & $0(0) c$ \\
\hline 19:0 cyclo w8c & $7.53(2.20) a$ & $9.65(0.33) a$ & $4.79(0.29) \mathrm{a}$ & $1.08(0.94) c$ & $4.35(0.20) \mathrm{b}$ & 10.0(1.14)a \\
\hline $18: 2 \omega 6 c(6,9)$ & 19.1(3.17)a & $8.09(0.88) b$ & $0.46(0.10) \mathrm{b}$ & $3.30(0.22) a$ & $3.65(0.38) a$ & $0.94(0.14) b$ \\
\hline $18: 3 \omega 6 \mathrm{c}(6,9,12)$ & $31.7(0.74) a$ & $6.70(0.50) \mathrm{b}$ & $0(0) \mathrm{b}$ & $5.63(0.42) a$ & $3.02(0.20) b$ & $0(0) c$ \\
\hline
\end{tabular}

ANOVA analysis was performed on all fatty acids present in abundance greater than $0.5 \mathrm{~mol} \%$. Values represent mean (standard deviation). Values in a given row followed by the same letter are not significantly different at $P \leqslant 0.05$ by one-way ANOVA and Tukey's HSD test. "Sum in Feature" are peaks identified in the MIDI software such that two adjacent peaks could not be distinguished or fully separated from each other.

specific steps in the FAME and Hybrid methods (Fig. 2). We asked whether certain steps are necessary when using the MIDI-FAME protocol on whole soil or soil extracts rather than microbial isolates.

First, we assessed the importance of using multiple hexane extractions to more thoroughly remove lipids from the acidic to organic phase following methanolysis (step 2 of FAME, and step 4 of Hybrid, Fig. 1). Not unexpectedly, lipid abundance decreased from the first extraction to the third (Fig. 5A and $5 B$ ). Overall lipid abundance using the Hybrid method decreased substantially (by an order of magnitude) after the first extraction. In contrast, when using the FAME protocol there was still a substantial amount of lipids extracted after three extractions. The numbers of individual lipids followed the same trend of marked decrease after the first extraction for the Hybrid protocol, with the number decreasing more slowly using the FAME method (Fig. 5C and 5D). It appears that having at least three extraction steps is important for the FAME protocol, but for the Hybrid protocol, two extraction steps will suffice.
Second, we tested the importance of the final basewashing and concentration steps shown in Fig. 1. Base washing is designed to remove contaminant lipids from the final product, and is recommended by MIDI, Inc., whereas the final concentration step is performed in PLFA, but is not part of the standard MIDI-FAME protocol (Fig. 1). We found that the base wash had little impact on the abundance and number of lipids extracted for both FAME and Hybrid methods, but the concentration step substantially increased the abundance and number of resulting lipids (Fig. 6). Therefore, the base wash step may not be necessary, especially when considering that each step adds time and potential loss of yield, to the procedure. The concentration step is likely to be important depending on the sensitivity of a researcher's given GC detector.

Overall, compared to FAME, the modified Bligh and Dyer (1959) two-phase aqueous-organic extraction with its final concentration step appears to remove the extractable lipids from soil humic materials (improving precision and purity) and increase lipid yields (improving detection limits). The third 

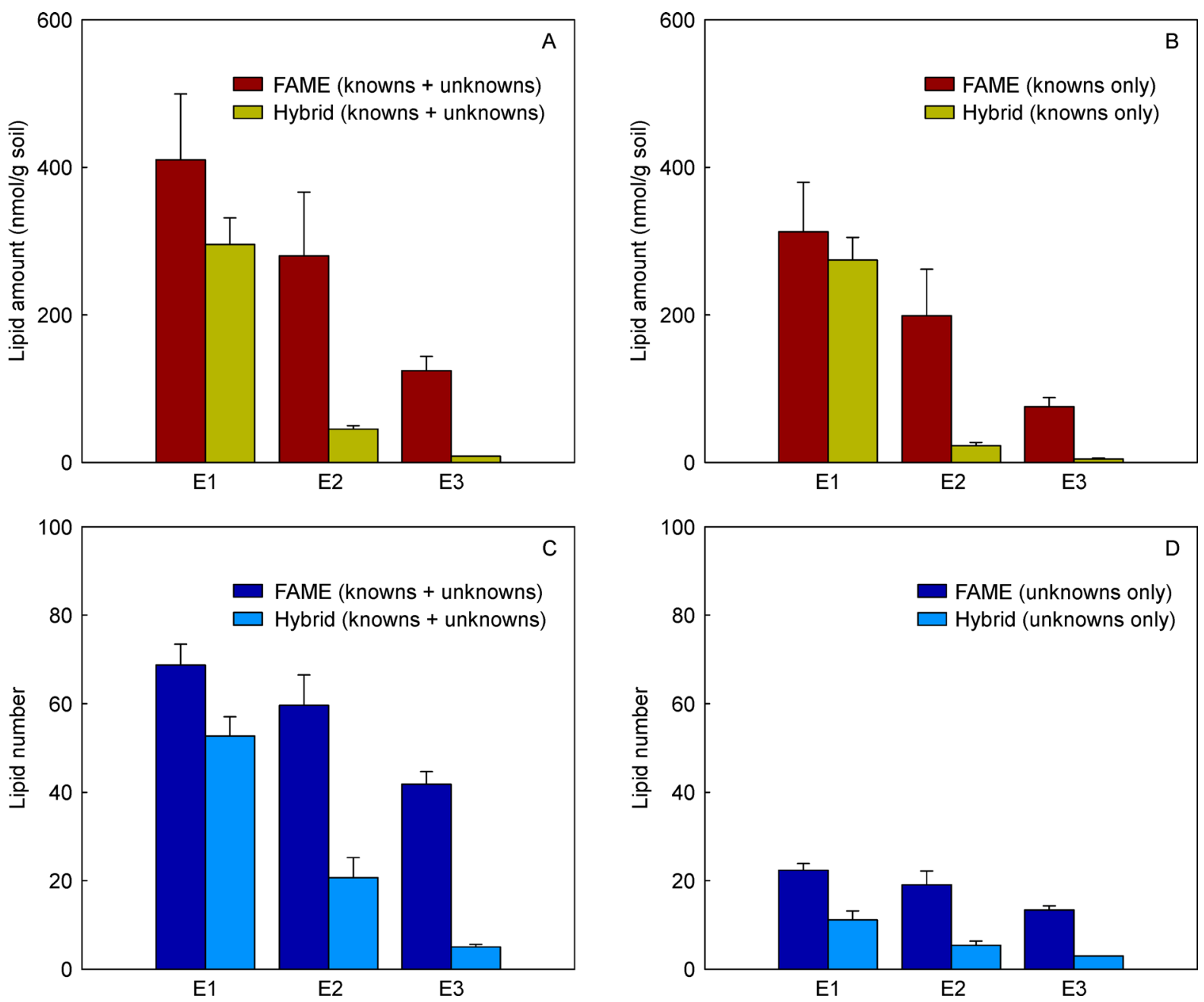

Fig. 5 The abundance (panels $A$ and $B$ ) and number (panels $C$ and $D$ ) of fatty acids in each successive extraction. A, the amount of total fatty acids extracted; $B$, the amount of known fatty acids extracted; $C$, the total number of fatty acids extracted; $D$, the number of unknown fatty acids extracted. E1, E2, and E3 represent the number of times of the hexane extraction, extraction 1, extraction 2, and extraction 3, respectively. Error bars represent one standard error of the mean.

method tested here, a Hybrid which combines properties of the PLFA and FAME methods, decreases sample processing time with minimal reduction in discriminatory power.

\subsection{Caveats}

While the hybrid lipid extraction protocol described here can greatly increase the use of lipid analysis and microbial analysis in ecosystem scale studies, there are caveats that should be considered with its use. First, because the lipid classes (phospholipids, neutral lipids, and glycolipids) remain combined in the FAME and hybrid methods, there is potential loss of physiologic information - such as membrane production or fungal lipid storage, that is available when analyzing those classes separately (Vestal and White, 1989; Bååth, 2003). On the other hand, a possible advantage of combining the lipid classes is that it may strengthen the power to detect the arbuscular mycorrhizal origin of the 16:1 $\omega 5 \mathrm{c}$ phospho- and neutral- lipids (Ngosong et al., 2012). Another consideration when using the Hybrid method is that while the number of unknown fatty acids (which could potentially be residual lipidlike soil molecules) were lower with the Hybrid than in the FAME protocol, the lower yield and lower number of unknowns in the PLFA method suggests that it provides more pure samples. If purity is a high priority, such as with ${ }^{13} \mathrm{C}$ PLFA analysis (Jin and Evans, 2010; Ruess and Chamberlain, 2010), the traditional PLFA method may be preferable.

\subsection{Conclusions}

In this paper we test a protocol designed to optimize and increase the throughput of lipid analysis by combining the first extraction step and last concentration step of PLFA, with the MIDI-FAME protocol. By performing the two-phase initial extraction and isolating the organic phase prior to performing FAME, the "Hybrid" protocol represents a balance between 

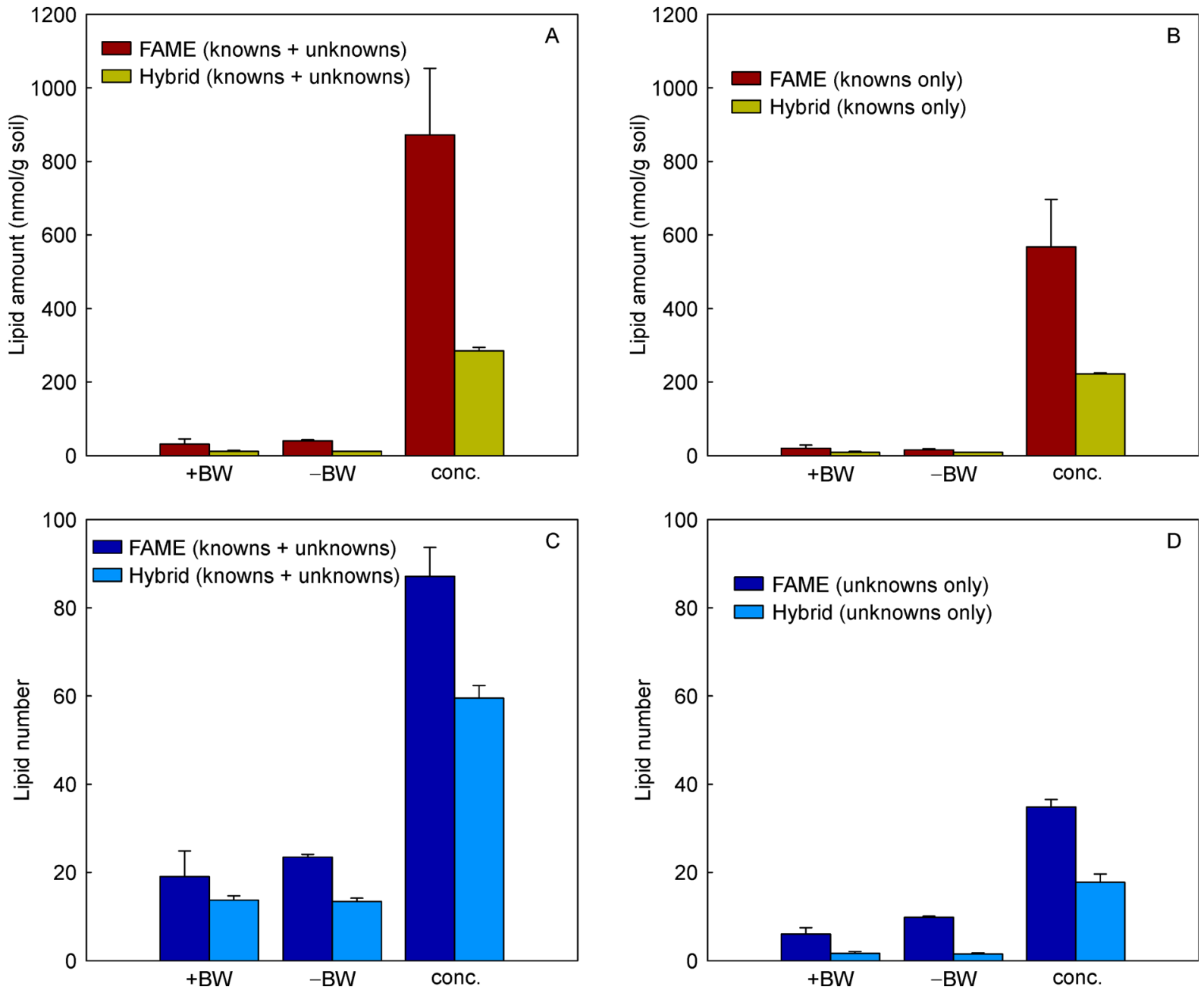

Fig. 6 The abundance (panels $A$ and $B$ ) and number (panels $C$ and D) of fatty acids with or without the base wash step or with the concentration step. A, the amount of total fatty acids extracted; $B$, the amount of known fatty acids extracted; $C$, the total number of fatty acids extracted; $D$, the number of unknown fatty acids extracted. $+B W=$ with the base wash step of the FAME procedure; $-B W=$ without the base wash step as after methylation in the PLFA procedure; and conc. = with the final concentration step of the PLFA procedure. The results of the concentration step were compared to the base wash test, as that test represents the methods without the final concentration step. Error bars represent one standard error of the mean.

speed and precision in fatty acid analysis. This method doesn't exactly replicate some aspects PLFA, but it has much less 'noise' than the FAME procedure. We have used the method successfully on a wide range of soils from tropical, to grassland, wetland, forest, boreal, and agronomic, to address and equally large range of research questions (Bartelt-Ryser et al., 2005; Fraterrigo et al., 2006; Mentzer et al., 2006; KaoKniffin and Balser, 2007; Smithwick et al., 2005; Ushio et al., 2008;Jokela et al., 2009; Gutknecht et al., 2012; Fichtner et al., 2014). This paper provides detail regarding the development of the method. Using this Hybrid method we are able to process significantly larger numbers of samples than in most traditional microbial ecology studies, with a lower standard deviation and coefficient of variation than with the FAME method. We conclude that this method represents a viable alternative to other lipid extraction protocols, or other microbial methods, when broad microbial data at a large scale is desired.

\section{Acknowledgments}

This study was supported by grants from the Kearney Foundation, the Andrew W. Mellon Foundation and the Department of Energy-Great Lakes Bioenergy Research Center DOE-GLBRC) funding. We would also like to thank the Matson laboratory (Stanford University) and Balser laboratory (University of Wisconsin-Madison) where method testing was performed. We are also grateful for the assistance of Carrie Nielsen and Phil Barak.

\section{Disclosure statement}

No potential conflicts of interest were reported by the authors. 


\section{References}

Baas-Becking, L.G.M., 1934. Geobiologie of Inleiding tot de Milieukunde. Van Stockum \& Zoon, The Hague.

Bååth, E., 2003. The use of neutral lipid fatty acids to indicate the physiological conditions of soil fungi. Microbial Ecology 45, 373 383.

Bailey, V.L., Peacock, A.D., Smith, J.L., Bolton, H. Jr, 2002. Relationships between soil microbial biomass determined by chloroform fumigation-extraction, substrate-induced respiration, and phospholipid fatty acid analysis. Soil Biology \& Biochemistry 34,1385 1389.

Balser, T.C., Firestone, M.K., 2005. Linking microbial community composition and soil processes in a California annual grassland and mixed-conifer forest. Biogeochemistry 73, 395-415.

Balser, T.C., Gutknecht, J.L.M., Liang, C., 2010. How will climate change impact soil microbial communities? In: Dixon, G.R., Tilston, E. (Eds.), Soil Microbiology and Sustainable Crop Production. University of Reading Press, Reading, U.K.

Balser, T.C., Kirchner, J.W., Firestone, M.K., 2002. Methodological variability in microbial community level physiological profiles. Soil Science Society of America Journal 66, 519-523.

Balser, T.C., Wixon, D.L., 2009. Investigating biological control over soil carbon temperature sensitivity. Global Change Biology 15, 2935-2949.

Bartelt-Ryser, J., Joshi, J., Schmid, B., Brandl, H., Balser, T., 2005. Soil feedbacks of plant diversity on soil microbial communities and subsequent plant growth. Perspectives in Plant Ecology, Evolution and Systematics 7, 27-49.

Ben-David, E.A., Zaady, E., Sher, Y., Nejidat, A., 2011. Assessment of the spatial distribution of soil microbial communities in patchy arid and semi-arid landscapes of the Negev Desert using combined PLFA and DGGE analyses. FEMS Microbiology Ecology 76, 492 503.

Bligh, E.G., Dyer, W.J., 1959. A rapid method of total lipid extraction and purification. Canadian Journal of Biochemistry and Physiology 37, 911-917.

Buyer, J.S., Sasser, M., 2012. High throughput phospholipid fatty acid analysis of soils. Applied Soil Ecology 61, 127-130.

Cavigelli, M., Robertson, G., Klug, M., 1995. Fatty acid methyl ester (FAME) profiles as measures of soil microbial community structure. Plant and Soil 170, 99-113.

Cavigelli, M.A., Robertson, G.P., 2000. The functional significance of denitrifier community composition in a terrestrial ecosystem. Ecology 81, 1402-1414.

Cusack, D.F., Silver, W.L., Torn, M.S., Burton, S.D., Firestone, M.K., 2011. Changes in microbial community characteristics and soil organic matter with nitrogen additions in two tropical forests. Ecology 92, 621-632.

Jesus, E., Da, C., Liang, C., Quensen, J.F., Suliwati, E., Jackson, R., Balser, T., Tiedje, J., 2016. Influence of corn, switchgrass, and prairie cropping systems on soil microbial communities in the upper Midwest of the United States. Global Change Biology. Bioenergy 8, 481-494.

Drenovsky, R.E., Elliott, G.N., Graham, K.J., Scow, K.M., 2004. Comparison of phospholipid fatty acid (PLFA) and total soil fatty acid methyl esters (TSFAME) for characterizing soil microbial communities. Soil Biology \& Biochemistry 36, 1793-1800.

Drijber, R.A., Doran, J.W., Parkhurst, A.M., Lyon, D.J., 2000. Changes in soil microbial community structure with tillage under long-term wheat-fallow management. Soil Biology \& Biochemistry 32, 14191430.

Fernandes, M.F., Saxena, J., Dick, R.P., 2013. Comparison of wholecell fatty acid (MIDI) or phospholipid fatty acid (PLFA) extractants as biomarkers to profile soil microbial communities. Microbial Ecology 66, 145-157.

Fichtner, A., von Oheimb, G., Härdtle, W., Wilken, C., Gutknecht, J.L. M., 2014. Effects of anthropogenic disturbances on soil microbial communities in oak forests persist for more than 100 years. Soil Biology \& Biochemistry 70, 79-87.

Fraterrigo, J.M., Balser, T.C., Turner, M.G., 2006. Microbial community variation and its relationship with nitrogen mineralization in historically altered forests. Ecology 87, 570-579.

Frostegård, A., Bååth, E., 1996. The use of phospholipid fatty acid analysis to estimate bacterial and fungal biomass in soil. Biology and Fertility of Soils 22, 59-65.

Frostegård, Å., Tunlid, A., Bååth, E., the Frostegård, 2011. Use and misuse of PLFA measurements in soils. Soil Biol Biochem 43, 1621-1625.

Gutknecht, J.L.M., Field, C.B., Balser, T.C., 2012. Microbial communities and their responses to simulated global change fluctuate greatly over multiple years. Global Change Biology 18, 22562269.

Haack, S.K., Garchow, H., Odelson, D.A., Forney, L.J., Klug, M.J., 1994. Accuracy, reproducibility, and interpretation of Fatty Acid methyl ester profiles of model bacterial communities. Applied and Environmental Microbiology 60, 2483-2493.

Herman, D.J., Firestone, M.K., Nuccio, E., Hodge, A., 2012. Interactions between an arbuscular mycorrhizal fungus and a soil microbial community mediating litter decomposition. FEMS Microbiology Ecology 80, 236-247.

Jackson, R., Fierer, N., Schimel, J., 2007. New directions in microbial ecology. Ecology 88, 1343-1344.

Jandl, G., Leinweber, P., Schulten, H.R., Ekschmitt, K., 2005. Contribution of primary organic matter to the fatty acid pool in agricultural soils. Soil Biology \& Biochemistry 37, 1033-1041.

Jin, V.L., Evans, R.D., 2010. Microbial 13C utilization patterns via stable isotope probing of phospholipid biomarkers in Mojave Desert soils exposed to ambient and elevated atmospheric $\mathrm{CO}_{2}$. Global Change Biology 16, 2334-2344.

Jokela, W.E., Grabber, J.H., Karlen, D.L., Balser, T.C., Palmquist, D. E., 2009. Cover crop and liquid manure effects on soil quality indicators in a corn silage system. Agronomy Journal 101, $727-$ 737.

Kao-Kniffin, J., Balser, T.C., 2007. Elevated $\mathrm{CO}_{2}$ differentially alters belowground plant and soil microbial community structure in reed canary grass-invaded experimental wetlands. Soil Biology \& Biochemistry 39, 517-525.

Kotas, P., Santruckova, H., Elster, J., Kastovska, E., 2018. Soil microbial biomass, acitivity and community composition along altitudinal gradients in the High Arctive (Billefjorden, Svalbard). Biogeosciences 15, 1879-1894. 
Liang, C., Fujinuma, R., Balser, T.C., 2008. Comparing PLFA and amino sugars for microbial analysis in an Upper Michigan old growth forest. Soil Biology \& Biochemistry 40, 2063-2065.

Liang, C., Gutknecht, J.L.M., Balser, T.C., 2015. Microbial lipid and amino sugar responses to long-term simulated global environmental changes in a California annual grassland. Frontiers in Microbiology 6, 385.

Mentzer, J., Goodman, R., Balser, T., 2006. Microbial response over time to hydrologic and fertilization treatments in a simulated wet prairie. Plant and Soil 284, 85-100.

Miura, T., Makoto, K., Niwa, S., Kaneko, N., Sakamoto, K., 2017. Compararision of atty acid methyl ester methods for characterization of microbial communities in forest and arable soil: Phospholipid fraction (PLFA) versus total ester linked fatty acids (ELFAME). Pedobiologia 63, 14-18.

Ngosong, C., Gabriel, E., Ruess, L., 2012. Use of the signature Fatty Acid $16: 1 \omega 5$ as a tool to determine the distribution of arbuscular mycorrhizal fungi in soil. J Lipids 2012, 236807.

Nielsen, P., Petersen, S.O., 2000. Ester-linked polar lipid fatty acid profiles of soil microbial communities: a comparison of extraction methods and evaluation of interference from humic acids. Soil Biology \& Biochemistry 32, 1241-1249.

Pankhurst, C.E., Yu, S., Hawke, B.G., Harch, B.D., 2001. Capacity of fatty acid profiles and substrate utilization patterns to describe differences in soil microbial communities associated with increased salinity or alkalinity at three locations in South Australia. Biology and Fertility of Soils 33, 204-217.

Pei, Z., Eichenberg, D., Bruelheide, H., Kröber, W., Kühn, P., Li, Y., von Oheimb, G., Purschke, O., Scholten, T., Buscot, F., Gutknecht, J.L. M., 2016. Soil and tree species traits both shape soil microbial communities during early growth of Chinese subtropical forests. Soil Biology \& Biochemistry 96, 180-190.

Schimel, J., 2004. Playing scales in the methane cycle: from microbial ecology to the globe. Proceedings of the National Academy of Sciences of the United States of America 101, 12400-12401.

Schmidt, J., Schulz, E., Michalzik, B., Buscot, F., Gutknecht, J.L.M., 2015. Carbon input and crop-related changes in microbial biomarker levels strongly affect the turnover and composition of soil organic carbon. Soil Biology \& Biochemistry 85, 39-50.

Schutter, M.E., Dick, R.P., 2000. Comparison of Fatty Acid Methyl Ester (FAME) methods for characterizing microbial communities. Soil Science Society of America Journal 64, 1659-1668.

Smithwick, E.A.H., Turner, M.G., Metzger, K.L., Balser, T.C., 2005. Variation in $\mathrm{NH}_{4}{ }^{+}$mineralization and microbial communities with stand age in lodgepole pine (Pinus contorta) forests, Yellowstone National Park (USA). Soil Biology \& Biochemistry 37, 15461559.

Tiedje, J.M., Asuming-Brempong, S., Nuslein, K., Marsh, T.L., Flynn, S.J., 1999. Opening the black box of soil microbial diversity. Applied Soil Ecology 13, 109-122.

Ushio, M., Wagai, R., Balser, T.C., Kitayama, K., 2008. Variations in the soil microbial community composition of a tropical montane forest ecosystem: Does tree species matter? Soil Biology \& Biochemistry 40, 2699-2702.

Vestal, J.R., White, D.C., 1989. Lipid analysis in microbial ecology: quantitative approaches to the study of microbial communities. Bioscience 39, 535-541.

Wallenstein, M., Hall, E., 2012. A trait-based framework for predicting when and where microbial adaptation to climate change will affect ecosystem functioning. Biogeochemistry 109, 35-47.

Willers, C., Jansen van Rensburg, P.J., Claassens, S., 2015a. Phospholipid fatty acid profiling of microbial communities-a review of interpretations and recent applications. Journal of Applied Microbiology 119, 1207-1218.

Willers, C., Jansen van Rensburg, P.J., Claassens, S., 2015b. Microbial signature lipid biomarker analysis- an approach that is still preferred, even amid various method modifications. Journal of Applied Microbiology 118, 1251-1263.

Zak, D.R., Holmes, W.E., White, D.C., Peacock, A.D., Tilman, D., 2003. Plant diversity, soil microbial communities, and ecosystem function: are there any links? Ecology 84, 2042-2050.

Zelles, L., 1999. Faty acid patterns of phospholipids and lipopolysaccharides in the characterization of microbial communities in soil: a review. Biology and Fertility of Soils 29, 111-129. 